\title{
Slow and fast light in optical fibres
}

\author{
The ubiquitous role of optical fibres in modern photonic systems has stimulated research to realize \\ slow and fast light devices directly in this close-to-perfect transmission line. Recent progress in \\ developing optically controlled delays in optical fibres, operating under normal environmental \\ conditions and at telecommunication wavelengths, has paved the way towards real applications
}

for slow and fast light. This review presents the state-of-the-art research in this fascinating field and

possible outcomes in the near future.

\section{LUC THÉVENAZ \\ Swiss Federal Institute of Technology, École Polytechnique Fédérale de Lausanne (EPFL), Institute of Electrical Engineering, Station 11, 1015 Lausanne, Switzerland e-mail: Luc.Thevenaz@epfl.ch}

The development of high-quality silica optical fibres with excellent transmission capabilities has directly contributed to the tremendous expansion of the global communication network. The success is due to their unrivalled low-loss performance (typically $50 \%$ of light is still present after a $15-\mathrm{km}$ transmission distance), their wide bandwidth, which allows terabits per second of information to be transmitted over more than $1,000 \mathrm{~km}$, and also their extremely low production cost. Realizing tunable delays for optical data signals directly in fibres, as well as integrating such devices into existing communication networks, is certainly a very attractive approach for optimizing the flow of data traffic in future networks. The approach may also enhance the capabilities of optical and microwave-on-optical-carrier signal processing. Given the limited possibilities for engineering the dispersion curve of standard single-mode fibres without creating complex photonic-crystal structures (see pages 448 and 465 of this issue $^{1,2}$ ), much research on fibre-based delays has been directed towards solutions based on spectral resonances to generate slow and fast light.

The initial pioneering demonstrations of slow and fast light in various media all exploited narrow spectral resonances, typically created by electromagnetically induced transparency ${ }^{3}$ or coherent population oscillation ${ }^{4}$. Narrow spectral resonances have a dramatic effect on optical propagation, as any sharp spectral change in the medium's transmission curve results in a steep quasi-linear variation of the effective refractive index with wavelength in the narrow spectral region near the resonance. This in turn results in a strong change in the group velocity at the exact centre of the resonance ${ }^{5}$. The velocity change is strongest for narrower spectral resonances (as explained in detail below) and for this reason, the early experiments were all carried out in special media, such as ultracold atomic gases or atomic transitions in a crystalline solid at a well-defined wavelength.

Within the resonance, the relationship between the change in the optical transmission and the delay can be determined as follows. The refractive-index change, $\Delta n$, as a function of the optical frequency, $v$, is calculated using the Kramers-Kronig transformation, and the index change associated with the group velocity, $\Delta n_{\mathrm{g}}$, is then found using the relationship $\Delta n_{\mathrm{g}}=\Delta n+v(\mathrm{~d} \Delta n / \mathrm{d} v)$ (ref. 5). Finally, the delay, $\Delta T$, added to the normal transit time in the medium is deduced from $\Delta n_{\mathrm{g}}$. Most spectral resonances can be described by a Lorentzian-shaped distribution, and in this important case the additional delay $\Delta T$ experienced by a signal placed at the centre of the resonance and spanning over a bandwidth well contained within the resonance is expressed as:

$$
\Delta T=\Delta n_{\mathrm{g}} \frac{L}{c}=\frac{G}{2 \pi \Delta v} \text { with } G=\gamma L,
$$

where $L$ is the propagation distance in the medium in metres, $c$ is the speed of light in a vacuum, $\Delta v$ is the full-width at half-maximum (FWHM) of the Lorentzian distribution in hertz and $\gamma$ is the peak value of the linear gain/loss coefficient at the resonance centre in $\mathrm{m}^{-1}$. It must be pointed out that $\gamma$ is positive in a gain situation, and so is $\Delta T$, implying the existence of signal retardation and the creation of slow light. In the case of absorption, $\gamma$ and $\Delta T$ are negative, and signal advancement is observed under fast-light conditions. Here, $G$ represents the total linear transmission - positive in the case of optical amplification and negative for absorption - and the signal power changes by a factor of $\mathrm{e}^{G}$ after propagation through the medium in the absence of saturation (small-signal regime).

Consequently, $\Delta T$ and changes in amplitude are closely related when using a single spectral resonance, indicating that creating delays with a flat amplitude response requires more sophisticated schemes. An important feature to acknowledge here is that for a gain or loss generated by optical pumping the amplitude of the coefficient $\gamma$ is a direct function of the pump power, and a fast and all-optical control of $\Delta T$ can be realized, in contrast with other technologies ${ }^{1,2,6}$. Equation (1) also shows that the delaying strength is dependent on the linewidth, $\Delta v$, of the resonance and $G$, and that the strongest slowand fast-light effect can be obtained in the centre of narrow and strong spectral lines. However the delay will only be effective for a signal with a spectral range that resides within the resonance; in other words the signal needs to be centred in the resonance and have a bandwidth smaller than $\Delta v$. Moreover, the signal bandwidth is further limited by the non-uniform transmission of a Lorentzian-shaped resonance; for example, in the situation of gain the spectral components of the signal near the centre of the resonance are favoured and have stronger transmission. This effect is further enhanced for high gains, as the power transmission depends exponentially on $G$, making the spectral range where the gain is nearly constant shrink as the gain increases. In most situations this effect manifests itself as a broadening of the optical pulses and sets a limit on the maximum delay $^{7,8}$. As a result, the spectral widths of the signal and resonance must be carefully adjusted and must be of the same order of magnitude for optimum performance. In practice the maximum delay only amounts to one 
to two pulse widths ${ }^{9}$, and breaking this deadlock remains a major challenge for research on slow light based on spectral resonances.

It should be noted that in the early experiments using atomic absorption lines a slow-light effect was obtained by creating a narrow transparent window within a spectral absorption line, using electromagnetically induced transparency $y^{3,10}$ or coherent population oscillation $^{4}$. In this case the absence of loss turns out to behave similarly to a gain resonance in terms of the slope of the refractiveindex spectral change, and in the centre of the transparency window the group velocity is thus reduced. Propagation in the transparency window offers the advantage of a minimal change in the signal amplitude, and this approach has been recently adopted in optical fibres by operating in the valley between two resonances, as described in a forthcoming section of this review.

The main obstacle for the creation of narrow spectral resonances (well below $100 \mathrm{GHz}$ ) in conventional glass optical fibres is related to the highly disordered nature of amorphous silica, a material that does not exhibit narrowband atomic transitions unless it is doped. The most efficient approach to create a narrowband gain or loss in optical fibres is to exploit an optical interaction that requires a strict phasematching condition to be satisfied. This way the waves interact only over the very limited spectral range where their amplitudes couple mutually and constructively. This can be realized using the nonlinear optical response of the material, where the energy from one optical wave is transferred to another optical wave. For instance, a resonant coupling over a narrow frequency range is observed in parametric interactions, where the generation of a third idler wave is required to satisfy the phase-matching condition. The optical wave that benefits from the energy transfer experiences a linear gain and a light-slowing effect, whereas the depleted wave will undergo a linear loss and a fast-light effect ${ }^{5}$.

This review describes the different approaches that have been demonstrated experimentally so far to generate slow and fast light directly in optical fibres. Owing to length limitations, this is not an in-depth, comprehensive review of the entire topic, but instead designed to offer non-specialists in the area valuable insights into the phenomenon. Special attention is paid to research using stimulated Brillouin scattering (SBS) to generate spectral resonances, as this is a topic that has been extremely active in recent years and has numerous benefits. In particular, the SBS approach is able to generate long delays at any wavelength, only requires a moderate pump power and is compatible with standard fibres and multigigabit-per-second data rates.

\section{APPROACHES FOR SLOW AND FAST LIGHT IN OPTICAL FIBRES}

\section{STIMULATED BRILLOUIN SCATTERING}

Among all the parametric processes observed in silica, SBS turns out to be the most efficient, as a result of the long lifetime of acoustic phonons that can efficiently grow and sustain the interaction. In its simplest configuration the coupling is between two optical waves, propagating in opposite directions in a single-mode fibre, and a longitudinal acoustic wave (stimulated by electrostriction), which plays the role of the idler wave in the interaction ${ }^{11}$. The interaction is efficient only if the two optical waves have a frequency difference that is resonant with the acoustic wave. This acoustic wave induces a dynamic Bragg grating in the fibre core that diffracts the light from the higher-frequency wave back into the wave with the lower frequency.

As a result of the slow velocity (around 5,800 $\mathrm{m} \mathrm{s}^{-1}$ ) of the acoustic wave compared with the speed of light in silica, the phase-matching criteria requires that the two lightwaves must counter-propagate, and phase matching is only possible under very strict conditions. An efficient conversion to the acoustic wave is observed only if the frequency difference between the optical waves is precisely set to a value known as the Brillouin frequency shift, $v_{\mathrm{B}}$, which is determined
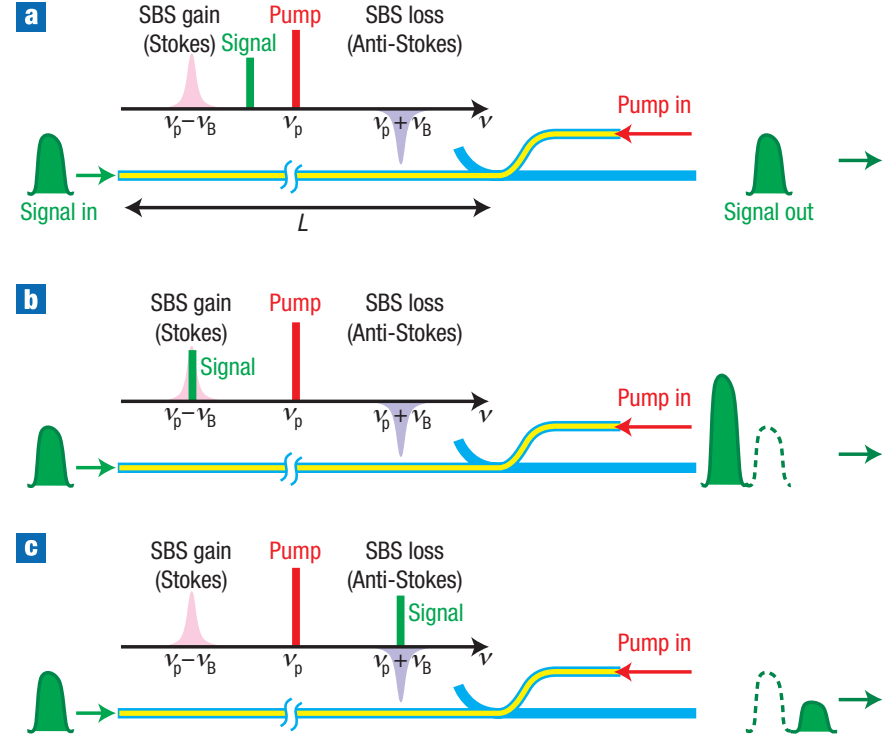

Figure 1 Principle of an all-optical fibre delay line based on SBS. a, The pump at optical frequency $v_{\mathrm{p}}$ is launched into the fibre from the far end so that it is counterpropagating with respect to the signal. When the signal frequency does not lie in the SBS interaction band, it travels through the fibre unaffected, with a delay given by its normal transit time and no amplitude change. $\mathbf{b}$, When the signal frequency is placed in the SBS gain resonance (Stokes), the signal is observed delayed with respect to its normal transit time (slow light). Simultaneously the SBS gain results in substantial signal amplification. c, When placed in the SBS loss resonance (anti-Stokes) the signal exits the fibre advanced with respect to its normal transit time and shows an attenuated amplitude (fast light).

by the acoustic velocity and the wavelength of the light in the fibre. The long lifetime of acoustic waves in silica (about $10 \mathrm{~ns}$ ) results in a very narrowband resonant coupling that translates into narrowband gain or loss for the interacting lightwaves. In optical fibres, the gain or loss spectral distribution faithfully follows a Lorentzian distribution, which is centred at the Brillouin frequency shift $v_{\mathrm{B}}(10-11 \mathrm{GHz}$ at $\lambda=1,550 \mathrm{~nm}$ ) and has a 30-MHz FWHM (ref. 12).

In practice, a very efficient narrowband amplification or attenuation can be created in any conventional silica single-mode fibre by propagating an intense monochromatic optical wave called the pump in the fibre, as shown in Fig. 1. A signal wave propagating in the opposite direction will experience a gain if its frequency is downshifted by $v_{\mathrm{B}}$ with respect to the pump (so-called Stokes band or wavelength) or a loss if it is upshifted by $v_{\mathrm{B}}$ to lie in the anti-Stokes band. An important property of the Brillouin interaction is that $\gamma$, introduced in equation (1), depends linearly on the pump intensity ${ }^{11,13}$ :

$$
\gamma=g_{\mathrm{o}} I_{\mathrm{p}}
$$

where $g_{0}$ represents the peak Brillouin linear gain in units of $\mathrm{m} \mathrm{W}^{-1}$ (around $2 \times 10^{-11} \mathrm{~m} \mathrm{~W}^{-1}$ in silica optical fibres) and $I_{\mathrm{p}}$ is the pump intensity in $\mathrm{W} \mathrm{m}^{-2}$. All other parameters in the interaction, such as $\Delta v$, do not depend on $I_{\mathrm{p}}$. Thus, it is clear by inserting equation (2) into equation (1), that $\Delta T$ naturally depends linearly on the pump power. This behaviour holds only if the pump experiences a negligible depletion during the interaction.

This linear dependence has important consequences for real systems $-\Delta T$ can be simply and optically controlled by varying the pump power. This linear response is certainly an essential asset for implementations in analog systems. The only practical difficulty 

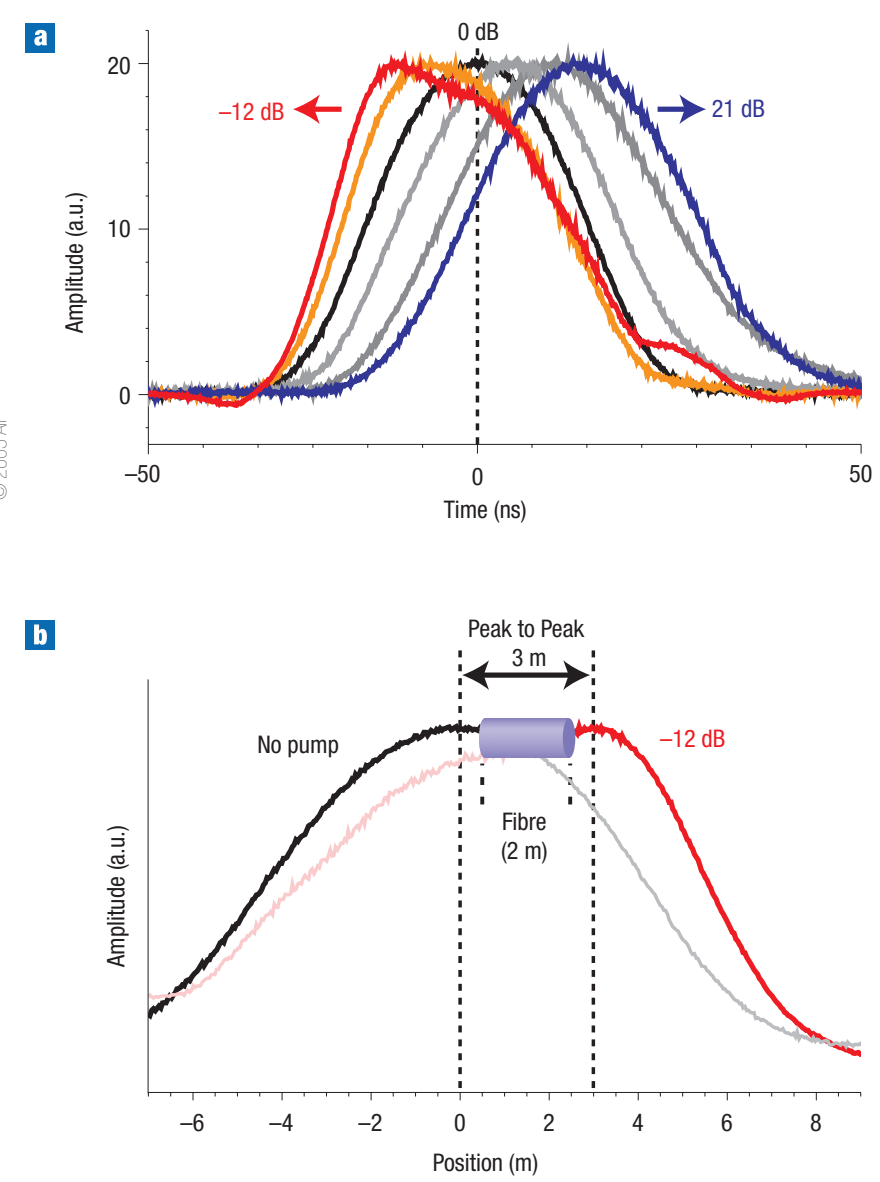

Figure 2 Pulse advancement and retardation through Brillouin-based slow- and fast-light effects in an optical fibre. a, Measured pulse delay (grey and blue curves) and advancement (red and orange curves) experienced by a 40-ns FWHM light pulse (black curve) propagating through a 2-m fibre. The time traces clearly show the distortion intimately related to the timing effect. Reproduced with permission from ref. 18. $\mathbf{b}$, Comparison between the respective positions of a pulse propagating in normal conditions, shown in black and grey ( $0-\mathrm{dB}$ gain: black curve in $\mathbf{a})$, and fastlight condition, shown in red and pink (-12-dB loss: red curve in a). In this situation, the pulse peak exits the fibre before entering it, corresponding to a negative group velocity. The shaded area represents the fibre length.

resides in the precise spectral positioning of the signal, as it must be accurately frequency-shifted with respect to the pump, with a typical precision and stability in the megahertz range.

Experimental solutions have been found to overcome this difficulty and the first demonstrations of SBS-induced slow and fast light ${ }^{14}$ in optical fibres were reported in 2005 initially at EPFL in Switzerland ${ }^{15}$ and independently a few months later at Cornell University in the USA (ref. 9). This research demonstrated that efficient delays can be realized using SBS. It also transpired that SBS in optical fibres is an excellent platform for testing the validity of the theoretical models describing slow and fast light, such as the theory of the perfect Lorentzian distribution of the spectral resonance. For instance, a predicted delay of $1 \mathrm{~ns}$ per $\mathrm{dB}$ gain was perfectly confirmed experimentally ${ }^{15}$ and changes in the propagation time of from $-8 \mathrm{~ns}$ in the fast-light regime up to $32 \mathrm{~ns}$ in the slow-light regime could be realized in these early demonstrations ${ }^{15}$. Some months later, higher effective gains were achieved by inserting spectrally neutral attenuators between fibre segments to prevent an excessive amplification of the signal while fully preserving the delaying effect ${ }^{16}$. In this way, delays could be extended up to 152 ns for a 40-ns input pulse, corresponding to a delay normalized to the pulse width of 3.6 (ref. 16). Nevertheless, as theoretically predicted ${ }^{8,9,17}$, the pulse experienced a very substantial broadening by a factor 2.4 for the maximum delay.

Even though impressive delays were obtained in these pioneering experiments they were achieved in kilometre-long fibres, and these delays represent only a minor fraction of the total transit time. The corresponding $\Delta n_{\mathrm{g}}$ is in the $10^{-3}-10^{-2}$ range, and the modification of the absolute group velocity is small, far from the amazing results obtained in atomic media. Comparable values for $\Delta n_{\mathrm{g}}$ (for the natural group index in silica, that is, 1.46) are possible in a fibre around $4 \mathrm{~m}$ long, but require a pump power of about $10 \mathrm{~W}$ to realize sufficient gain. An experiment with a 2 -m-long fibre ${ }^{18}$ slowed the signal down to a velocity of $71,000 \mathrm{~km} \mathrm{~s}^{-1}$, corresponding to a group index of 4.26 or a speed reduction by nearly a factor of three using a pump power estimated to be around $5 \mathrm{~W}$.

Using the same experiment researchers at EPFL in Switzerland were also able to drastically accelerate the signal in a fast-light configuration using the Brillouin-loss process (Fig. 2), and in fact the signal could reach the regime of superluminal propagation, namely a group velocity that exceeds the velocity of light in a vacuum. The maximum temporal advance attained was $14.4 \mathrm{~ns}$, with respect to a 10 -ns normal propagation time in the $2-\mathrm{m} \mathrm{fibre}^{18}$. In this superluminal propagation regime of negative group velocity, the signal effectively exits the fibre before entering it. This can be visualized in Fig. $2 b$, where it can be clearly seen that the leading edge of the pulse is reshaped, so that the peak of the pulse is apparently simultaneously present at the input and the output of the fibre. This situation is of course possible only at the expense of a severe distortion of the pulse to satisfy all the principles given by causality and relativity for the transfer of information, as fully and elegantly described by Leon Brillouin in the 1910's (refs 19-21). These results were the first demonstration that a negative group velocity can actually be achieved in optical fibres.

The ability to realize delays in a short fibre is not only a scientific curiosity, but has an important impact on practical applications, as the reconfiguration time for the delays is basically given by the transit time through the delaying fibre. As the total gain is determined by the product of the pump power and the fibre length, shortening the fibre requires increasing the pump power in the same proportion to maintain the same gain and thus the same $\Delta T$. Using a pump power of many watts limits the practicability, and much research effort has been dedicated to developing fibres in materials with a larger natural Brillouin gain. One of the first attempts was using bismuth oxide optical fibres $^{22}$, which gave a significant improvement compared with silica fibres ${ }^{18}$ - a fivefold reduction of the group velocity in a 2-m fibre using just $400 \mathrm{~mW}$ of continuous-wave pump power. An even higher efficiency was obtained by using $\mathrm{As}_{2} \mathrm{Se}_{3}$ chalcogenide fibres ${ }^{23}$, for which a 46-ns delay was realized in a 5-m fibre using only $60 \mathrm{~mW}$ of pump power ${ }^{24}$. For a better comparison these results can be reformulated in terms of comparative lengths: using the same pump power, 1 metre of chalcogenide fibre will show the same delay as 4 metres of bismuth oxide fibre or 110 metres of standard silica fibre. Of course using such exotic glass fibres is only practical for short delay segments as they normally exhibit an attenuation far exceeding that of silica fibres.

However, despite these impressive achievements obtained using simple benchtop set-ups, it was soon identified that the narrowband nature of SBS makes it unsuitable for broadband data communications, as the $30-\mathrm{MHz}$ linewidth of the gain spectral distribution restricts the full delaying effect to pulses longer than $40 \mathrm{~ns}$. This limitation stimulated research towards investigating different gain processes that have a larger intrinsic linewidth.

\section{STIMULATED RAMAN SCATTERING}

It was soon demonstrated that stimulated Raman scattering (SRS), another parametric process in fibres that involves material vibration, 
can also create the required conditions for slow light propagation ${ }^{25}$. In fact optical phonons have an essentially zero velocity, thus automatically satisfying the phase-matching criteria. As a result of the structural disorder in silica the gain process is naturally broadband and is therefore widely used in modern telecommunication networks for amplification. The results in ref. 25 evaluate the interaction bandwidth to be $3,000 \mathrm{GHz}$ and demonstrate delays of up to $370 \mathrm{fs}$ for ultrashort pulses of $430 \mathrm{fs}$ duration. In addition to a smaller natural gain than SBS, the ultrawide bandwidth of this parametric process further reduces the strength of the delay and SRS turns out to offer valuable fractional delays only for subpicosecond pulses, for which it is probably the most efficient approach.

\section{OPTICAL PARAMETRIC AMPLIFICATION}

To achieve a better match between the gain spectral linewidth and the bandwidth of multigigabit-per-second signals, another approach using the process of parametric amplification based on the Kerr optical nonlinearity has been proposed ${ }^{26,27}$. In this process the idler wave is another optical wave and the three waves involved in the interaction are co-propagating. They must obey a phase-matching condition that is more rigorous than for SRS, but less strict when compared with SBS and its counter-propagating-wave requirement. In addition, the linewidth of the interaction can be widely tuned by carefully placing pump and signal wavelengths within the fibre dispersion curve. In this way, delays of up to 160 ps using 70-ps pulses were obtained in a 2-km fibre using $1 \mathrm{~W}$ pump power, with a bandwidth capability of many tens of gigahertz (ref. 26). Despite the lower efficiency of this parametric process when compared with stimulated scattering (SBS and SRS), this approach offers the best natural delaying capability for bandwidths of tens to hundreds of gigahertz.

\section{SPECIAL-FIBRE APPROACHES}

Finally, two other approaches that are not based on a parametric interaction have been proposed, but these require special or processed fibres. The first technique exploits the coherent population oscillation in a fibre doped with erbium ions, where a very narrow resonance is created with a bandwidth essentially determined by the erbium lifetime (around $10 \mathrm{~ms})^{28}$. Large delays up to a fraction of a millisecond can be obtained, but with only a moderate bandwidth in the kilohertz range. As a result, this approach is of limited use for real signal-processing applications, but is a good tool for investigating large velocity changes. An interesting study of the propagation in a negative-velocity regime under extreme fast-light conditions was performed using this approach, with step-by-step observation of a backward propagating pulse ${ }^{29}$.

The second technique uses so-called gap solitons that can be observed on the edge of the reflection band of a Bragg grating imprinted in a fibre, where the dispersion curve shows sharp transitions $s^{30}$. Simulations show that delays of up to 20 pulse widths using subnanosecond pulses can be obtained in a 100 -mm-long grating with minor amplitude changes and low distortion. Delay tunability is achieved by simultaneously adjusting the launch power and the wavelength detuning between the soliton and the grating band edge. Such a delaying scheme is restricted to soliton-shaped pulses, requiring a fixed pulse width for a given peak power that normally lies in the kilowatt range. However, the approach is very attractive and may have an important impact on practical applications, as large delays can be obtained over a short distance and with a minor amplitude change.

All these approaches face important challenges before they can enter into the field of real applications. For instance, the ultrabroadband nature of Raman scattering in optical fibres results in a poor delaying efficiency at bandwidths compatible with practical data-transmission systems, and so far no further developments were reported since the first publication ${ }^{25}$. Optical parametric amplification based on the Kerr effect is very sensitive to the fluctuations of the chromatic dispersion along the fibre, which cause an uncontrolled broadening and distortion of the gain spectral distribution ${ }^{27}$.

As far as Brillouin scattering is concerned, a simple solution has now been discovered to overcome the very narrowband nature of this interaction. This solution is based on the unique property that Brillouin spectral resonances produced by different laser lines can be combined and superposed to result in an enlarged spectral distribution. The laser lines can be generated by distinct lasers or as the result of simple modulation. This unique feature of SBS, which will be described in more detail in the following section, overcomes the bandwidth hurdle that was blocking the use of SBS in real applications, and has given rise to numerous reports of advanced schemes realizing functions that would be very difficult to achieve using other interactions.

\section{EXTENDED PERFORMANCE USING PUMP MODULATION}

The linear dependence of the SBS gain on pump power has a crucial impact on the design of delay schemes - if the pump power spectrum is polychromatic, it can be decomposed into a sum of monochromatic waves. Each of these waves produces its own SBS spectral resonances, which have delaying effects for the fraction of the signal spectrum that matches its narrowband resonance. The overall effect on the signal will be the summation of all these separate and partial contributions. This situation can be represented by a fictitious monochromatic pump stimulating a fibre medium, and showing an effective Brillouin gain spectral distribution $g_{\mathrm{B}}^{\text {eff }}(v)$. The gain spectral distribution corresponds to the convolution of the natural narrowband Brillouin gain spectrum $g_{\mathrm{B}}(v)$, with the normalized pump power spectrum

$$
g_{\mathrm{B}}^{\text {eff }}(v)=g_{\mathrm{B}}(v) \otimes \frac{P_{\text {pump }}(v)}{P_{\text {pump }}^{\text {tot }}} \text { with } P_{\text {pump }}^{\text {tot }}=\int_{0}^{\infty} P_{\text {pump }}(v) \mathrm{d} v
$$

where $\otimes$ conventionally denotes the convolution operator, $P_{\text {pump }}(v)$ the pump-power spectral density in $\mathrm{W} \mathrm{Hz}^{-1}$ and $P_{\text {pump }}^{\text {tot }}$ is the total pump power in $\mathrm{W}$.

\section{MODULATION USING A DISCRETE LINE SPECTRUM}

The relationship between the effective Brillouin gain spectral distribution and the pump-power spectral density (Fig. 3) has a tremendous impact on applications for signal transformation through SBS. It means that the gain spectral distribution can be drastically modified and shaped by the pump power spectrum. This is depicted in Fig. 3a, showing that the result of the convolution is to smooth the actual pump power spectrum. In fact $g_{\mathrm{B}}^{\text {eff }}(v)$ is essentially given by the pump power spectrum when it is much broader than the natural Brillouin resonance (around $30 \mathrm{MHz}$ at $\lambda=1,550 \mathrm{~nm}$ ).

The ability to modify the SBS gain spectrum was soon identified after the pioneering experiments on SBS delaying, and the first demonstration was realized using the simplest polychromatic spectrum made of just two frequency components ${ }^{31}$. The result of the convolution is to produce a double resonance, and if the peak separation is comparable to the natural Brillouin linewidth, the overlapping of the resonances generates a reverse slope and fast light can be realized in a gain regime ${ }^{32}$. This situation is depicted in Fig. $3 c$, showing the additional advantage that the delaying effect is produced with a much reduced signal amplification. It turns out that the signal reduction has a limited advantage, as a large gain is still present in the centre of the two resonance peaks, which may amplify the spontaneous emission and limit the maximum pump power for generating the delays. This cannot be solved by producing slow light in the loss regime ${ }^{33}$, as gain resonance peaks will also be generated symmetrically in the Stokes band.

But this demonstration clearly shows that these new schemes of pump modulation can be used to slow light. In the particular case 

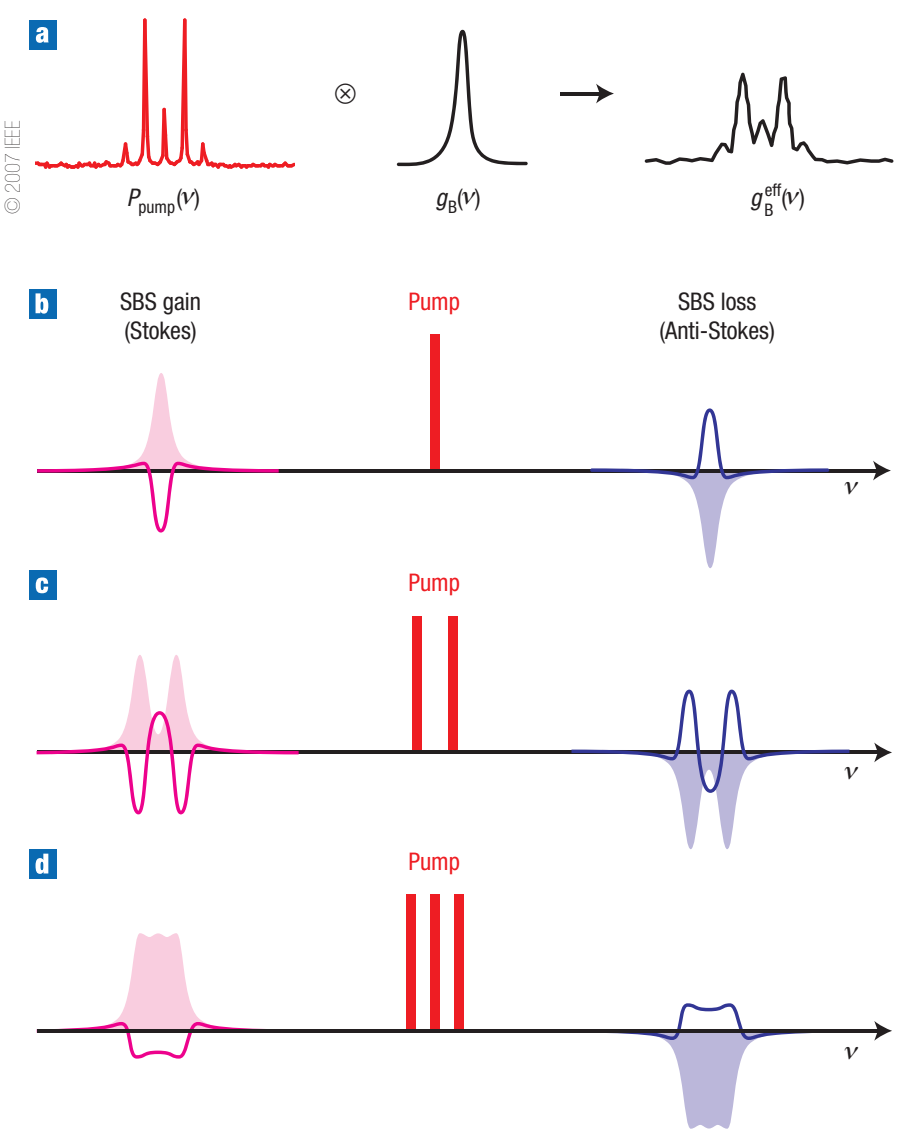

e

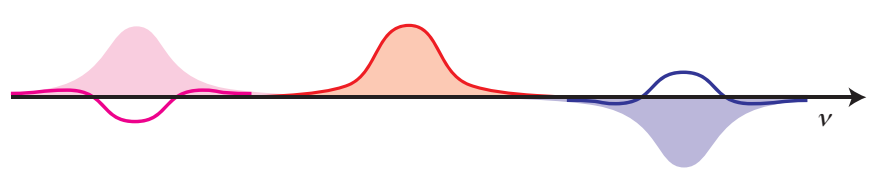

$\mathbf{f}$

Pump

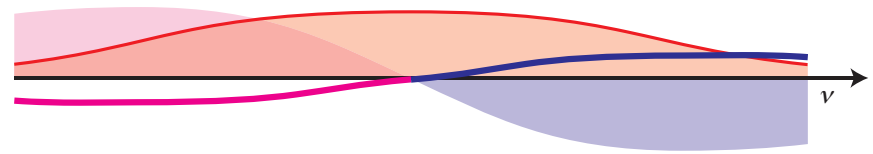

Figure 3 Examples of slow- and fast-light schemes based on SBS using natural and synthesized gain spectral distribution. a, The effective Brillouin gain spectrum $g_{B}^{\text {eff }}(v)$ is given by the convolution of the pump-power spectral distribution $P_{\text {pump }}(v)$ and the intrinsic Brillouin gain spectrum $g_{B}(v)$, offering the possibility of synthesizing tailored gain spectral distributions. Reprinted with permission from ref. 67. b. Slow- and fastlight obtained using the intrinsic SBS resonance $g_{B}$ and a monochromatic pump. The gain and loss spectral distributions are represented by a solid line. This change peaks at the centre of the Lorentzian resonances. c, A bichromatic pump. The overlapping of the two SBS resonances causes an inversion of the curvature at the centre of the spectral distribution, which turns into fast light in the gain regime and slow light in the loss regime with a reduced amplitude change. $\mathbf{d}$, A trichromatic pump. A relatively flat gain spectrum can be synthesized, which results in an enhanced delaying effect and a reduced distortion. e, A homogeneously broadened pump. The shape of the gain spectral distribution is a replica of the pump spectrum, and broadband delays can be generated at a bandwidth far exceeding that of the natural Brillouin resonance. $\mathbf{f}$, An extreme case of a broadened pump showing a bandwidth exceeding the Brillouin frequency shift $v_{B}$. The SBS gain and loss spectra overlap and mutually cancel around the pump frequency, placing a boundary at $v_{\mathrm{B}}$ for the maximal gain broadening that can be synthesized. of the two-frequency pump, the delay can be continuously tuned by changing the frequency separation between the two spectral lines instead of varying the pump power, and may even enable switching from slow to fast light propagation ${ }^{31}$.

Modifying the actual spectral shape of the resonance is also interesting for the optimization of the delay effect. For instance an index slope steeper than observed for a natural Lorentzian distribution can be produced within the same bandwidth by a properly shaped gain spectrum. In addition, this reshaping can also significantly reduce the distortion experienced by the signal. Through this approach, interesting studies were carried out by varying the relative amplitude and the frequency separation between evenly spaced frequency components and the best slow-light response was obtained for a three-tone pump spectrum with identical amplitudes for each line $e^{34,35}$, as depicted in Fig. 3d.

\section{MODULATION USING A CONTINUOUS BROADBAND SPECTRUM}

Nevertheless, all these experiments, which use a moderate number of discrete lines in the pump spectrum, do not completely overcome the limitation caused by the narrowband nature of SBS. However, the effective Brillouin gain spectrum can be continuously broadened using a randomly modulated pump source ${ }^{36}$, as depicted in Fig. 3e. A broadened smooth SBS gain spectrum with a FWHM of up to $325 \mathrm{MHz}$ was generated by directly modulating the current of the pump laser diode using a pseudo-random bit sequence at $38 \mathrm{Mbit} \mathrm{s}^{-1}$. The width of the pump spectrum is basically controlled by the amplitude and the bit rate of the modulating signal. The bit rate is set high enough to ensure that the pump spectrum seen by the counter-propagating pulse is homogeneous when integrated over the entire fibre length $(6.7 \mathrm{~km}$ or $33 \mu$ s transit time) and that the pump time sequence does not therefore modulate the signal. Pulses as short as $2.7 \mathrm{~ns}$ could be delayed in this effective gain spectrum in a process formally identical to a natural resonance, but with a linewidth of the gain resonance that can potentially be arbitrarily extended.

This extension of the bandwidth has definitely removed a bottleneck that was previously considered impossible to overcome using the SBS approach. However, it must be pointed out that this bandwidth extension requires that the pump power is raised proportionally to the amount of spectral broadening to maintain the same normalized delay, and proportionally to the square of the relative spectral broadening to keep the same absolute delay. This is because on one hand the peak effective SBS gain is decreased proportionally to the broadening as a result of the convolution, and on the other hand the slow-light delay is inversely proportional to the resonance linewidth, as shown in equation (1).

Detailed studies of the broadband SBS delays have been conducted where the pump spectrum was broadened using an electrical noise source superimposed on the injection current of the pump laser diode $\mathrm{e}^{37}$. Pulses $75 \mathrm{ps}$ in duration could be delayed, proving convincingly that this type of delay line is compatible with a data rate of up to $10 \mathrm{Gbit} \mathrm{s}^{-1}$. The SBS gain spectrum could be broadened up to the point where the Stokes and anti-Stokes bands start to overlap and start to mutually neutralize, as shown in Fig. 3f. This effect prevents any further extension of the gain linewidth and can be seen as the limit to the actual broadening. This corresponds to an equivalent gain bandwidth of $12.6 \mathrm{GHz}$, which can be considered the maximum bandwidth obtained by SBS and a single broadened pump. In fact this limit can be overcome by using multiple pumps.

The effective SBS gain spectra obtained using the pseudorandom bit generator or the noise source provide typical bell-shaped distributions that turn out to be not ideal as far as the phase gradient through the gain resonance and the signal distortion are concerned. An interesting implementation was realized in ref. 38, in which the gain spectral distribution was shaped to maximize the phase gradient 
and thus the delay strength. The spectrum of a pump semiconductor laser was tailored using a combination of a deterministic, periodic current modulation together with a small random component to obtain a continuous effective SBS gain spectrum with sharp edges. For equal pump powers and gain bandwidths, such a tailored gain spectrum introduces 30-40\% longer delays than a standard Lorentzian resonance. This approach was further developed to optimize both the delay and the distortion, and it was found that the ideal gain spectral distribution was approximately square-shaped ${ }^{8,39-41}$.

Extending the bandwidth is done at a serious cost in terms of pump power. At $10 \mathrm{GHz}$, an equivalent fractional delay to that obtained using the natural non-broadened Brillouin resonance requires 350 times more pump power. Considering that the minimal pump power to obtain a fractional delay of 1 (one pulse width) is $5 \mathrm{~mW}$ in very long fibres using the natural resonance, this would bring the required pumping level to $1.75 \mathrm{~W}$ and above to secure a suitable delay at $10 \mathrm{Gbit} \mathrm{s}^{-1}$. As recently demonstrated ${ }^{42}$, this power requirement can be substantially reduced when a real bit stream is considered, as the signal spectrum is essentially made of evenly spaced lines showing a moderate broadening. Efficient timing control can be obtained using a pump comb spectrum, spectrally concentrating the power where the signal has most of its frequency content. In addition the delay can be controlled by keeping the pump power constant and slightly shifting the comb frequency with respect to the data repetition frequency, providing a flexible way to shift between slow- and fast-light effects. The main restriction of this scheme is the loss of versatility, as prior knowledge of the signal characteristics must be known and the delay line is designed for a very specific type of signal.

\section{ADVANCED DELAY SCHEMES}

Stimulated Brillouin scattering offers an additional degree of freedom through the superposition of gain and loss spectral distributions. This can be realized by using two pumps separated by twice the Brillouin frequency shift $v_{\mathrm{B}}$ (around $21-22 \mathrm{GHz}$ at $\lambda=1,550 \mathrm{~nm}$ ), in which case the Brillouin loss spectrum (anti-Stokes band) of the lowerfrequency Pump 1 is superposed on the Brillouin gain spectrum (Stokes band) of the higher-frequency Pump 2, as shown in Fig. 4a-c. This possibility offers new functionalities through the complete or partial overlapping of gain and loss spectral distributions with either identical or different broadening.

This feature was exploited to further extend the bandwidth of an SBS delay line over the $12-\mathrm{GHz}$ limit provided by a single pump laser ${ }^{43}$. Two pumps were exactly separated by twice the Brillouin frequency shift $v_{\mathrm{B}}$ and identically broadened, so that the loss spectrum of Pump 1 is perfectly cancelled by the gain spectrum of Pump 2 at any frequency. This way the gain spectrum of Pump 1 is no longer compensated by its own loss spectrum, and the effective gain broadening can be extended until the loss spectrum of Pump 2 starts to overlap the gain spectrum of Pump 1. The situation is depicted in Fig. 4c. The limit of the effective gain linewidth can be actually doubled to reach $25 \mathrm{GHz}$. Pulses with a duration of 37 ps were delayed up to $10.9 \mathrm{ps}$, as shown in Fig. 4d, and the effective gain spectral distribution was measured to be approximately Gaussian with an estimated linewidth of $27 \mathrm{GHz}$ (ref. 43).

This scheme can be further extended by adding another broadened pump separated from Pump 2 by the Brillouin shift $v_{\mathrm{B}}$ to compensate the loss spectrum of Pump 2. This extends the bandwidth by another $12 \mathrm{GHz}$ to get close to the $40-\mathrm{GHz}$ limit needed for ultrahigh-datarate optical communications. The bandwidth can thus be increased in $v_{\mathrm{B}}$ steps by increasing the number of pumps. However, this raises a serious practical difficulty, as such a scheme would require a massive broadening of the pump's emission spectrum, with the consequence that the power of each individual pump must also be increased in the same proportion to maintain the fractional delaying strength.
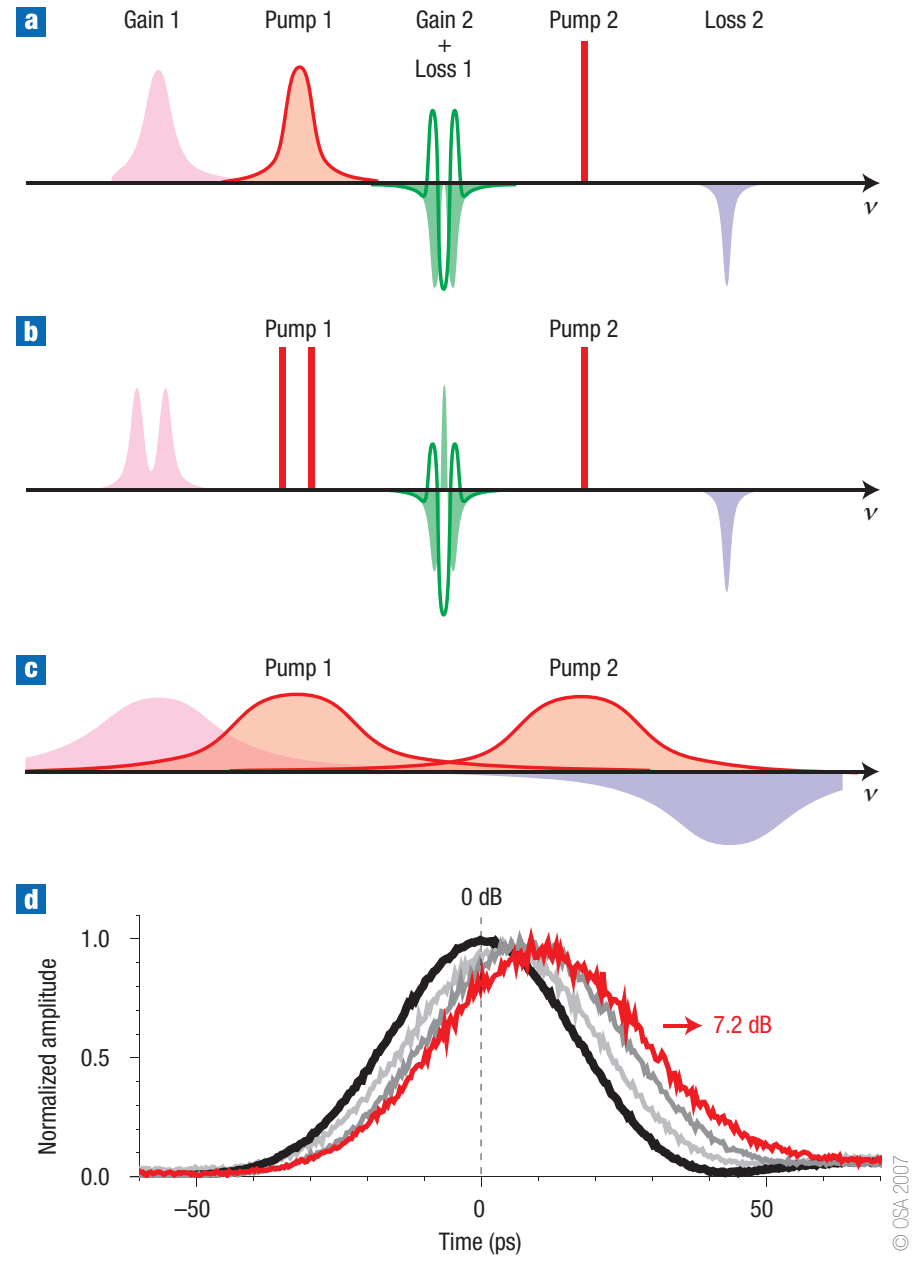

Figure 4 Advanced delay schemes obtained through the superposition of SBS gain and loss spectra generated by two distinct pumps separated in frequency by twice the Brillouin shift $v_{\mathrm{B}}$. The resulting gain spectral distribution mid-positioned between the two pumps is shown shaded in green and the corresponding group velocity change by a solid green line. a, Zero gain delays are obtained by using two pumps with substantially different broadening. The peak gains and losses created by the two pumps are identical and cancel at the centre of their overlapping range. $\mathbf{b}$, Enhanced delaying with the same amplification can be obtained by superposing the spectra of a monochromatic and a bichromatic pump. c, Two widely broadened pumps with identical bandwidth make their gain and loss spectra exactly compensate in the medium-frequency range. The loss of Pump 2 is thus compensated by the gain of Pump 1 and no longer cancels the gain of Pump 2, which can be further broadened by up to twice the Brillouin shift $v_{B}$. d, Time evolution of 37-ps pulses delayed by an effective SBS gain spectrum showing an equivalent linewidth of $25 \mathrm{GHz}$. The maximum delay is 10.9 ps for a 7.2-dB gain. Reprinted with permission from ref. 43.

The superposition of gain and loss spectra of different spectral widths also offers the possibility of achieving slow and fast light in a fully transparent regime. A transmission spectrum similar to that obtained by electromagnetically induced transparency can be synthesized, as a narrowband gain can open a transparency window in a broadband loss spectrum. This situation is depicted in Fig. $4 \mathrm{a}$ and clearly shows that the gain compensation maintains the sharp spectral transitions necessary to induce slow and fast light propagation, as experimentally demonstrated in ref. 44 . Figure 5 shows that delays similar to those obtained using a single monochromatic pump are achieved, but with less than $1-\mathrm{dB}$ variations in the signal intensity 


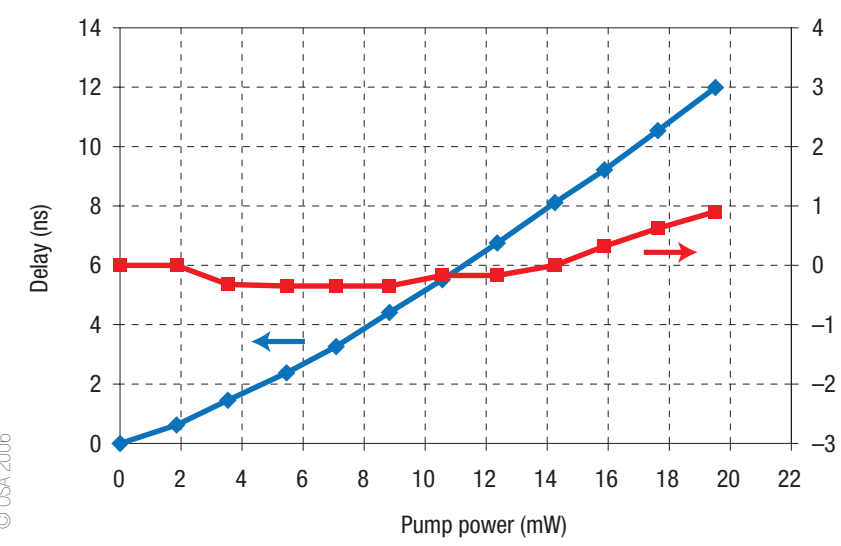

Figure $\mathbf{5}$ Experimental demonstration of the generation of efficient delays with a minor signal intensity change. The induced delays are shown in blue as a function of the narrow-linewidth pump power, and the corresponding intensity changes are shown in red, for a signal propagating in the middle of the spectral feature depicted in Fig. 4a. Reprinted with permission from ref. 44.

over the entire 12-ns delay range. Signal advancement using fast light was also observed by simply swapping the pumps' spectral positions. This result has an important impact on practical applictions, as any change in the signal intensity remains an unwanted side effect of the delay process. It must be pointed out that the physical nature of the transparency in this case is entirely different to electromagnetically induced transparency and in this gain-loss compensation scheme the signal unfortunately contains all the excess noise resulting from the amplification process.

Following the same approach, gain compensation by a superimposed broadband loss or by two narrowband losses placed on the wings of the gain spectral distribution, as shown in Fig. 4b, offers the possibility of enhancing the delaying capability by up to a factor of two when compared with a single-resonance scheme ${ }^{45}$.

Recently, self-generated fast light, in total absence of any pump source, has been reported ${ }^{46}$. In this implementation, the signal is amplified above the critical Brillouin power, often named the Brillouin threshold, so that all the signal light is converted into a backwards propagating wave resulting from amplified spontaneous emission. This intense light is spectrally positioned below the signal frequency by exactly the fibre Brillouin shift, and a Brillouin loss resonance exactly positioned at the signal frequency is created. In a $12-\mathrm{km}$ fibre temporal advancements of up to $12 \mathrm{~ns}$ were continuously generated by varying the signal input power between $10 \mathrm{~mW}$ and $630 \mathrm{~mW}$. This implementation offers many practical advantages in addition to its inherent simplicity, such as a constant signal amplitude at the exit of the delay line and the interesting property that the spectrum of the amplified Brillouin emission matches the signal spectral distribution. In other words, it allows the SBS effective loss spectrum to selfadapt to the signal spectral distribution and automatically match its bandwidth.

\section{PERSPECTIVES}

Slow- and fast-light delay lines in optical fibres based on SBS show unprecedented flexibility, such as the ability to shape the spectral resonance, which is made possible through the modulation of the pump. In recent years, attractive solutions have been proposed to overcome the challenges associated with use in real applications. These solutions include the realization of broadband delays of up to $25 \mathrm{GHz}$ and more, optimization of the gain spectral distribution for signal delays with minimal distortion, the achievement of delays in metre-long fibres and the realization of delays with no amplitude change.

However, as pointed out by many valuable studies ${ }^{7,47-49}$, the storage capability of slow- and fast-light delay lines based on amplifying spectral resonances, such as those presented in this review, are still far below the expectations for optically buffering a bit sequence. A simple analysis shows that storing a bit sequence of more than five bits remains unrealistic ${ }^{8,50}$. Moreover the performance required by modern telecommunication systems and optical digital processing in terms of delay and bandwidth would require a tremendous pumping power, which is arguably not practical ${ }^{48}$. Although other technologies, such as coupled resonators ${ }^{6,50}$, potentially provide improved storage capabilities, the all-optical and fast delay control that characterizes the fibre-based techniques is not yet possible in such structures. This means that it is difficult for slow- and fast-light delay lines to meet the target application that motivated the early research efforts, that is, the all-optical buffering required for future all-optical routers ${ }^{51}$. For such an application, wavelength conversion associated with a dispersive optical line has proven to be an efficient solution, and the delaying of a full sequence of 1,000 bits at $10 \mathrm{Gbit} \mathrm{s}^{-1}$ has already been demonstrated ${ }^{52-54}$. Coherent optical storage also appears to be a promising approach, and recent results show that SBS can realize this storage function in optical fibres ${ }^{55}$. This conclusion has pushed the research community to reconsider the potential applications and strengths of each approach.

Recently, retiming applications in digital transmissions requiring an error-free one-bit delay have been investigated, and SBS slowlight fibre delay lines have proven to be a robust solution ${ }^{56}$. For an analog signal, it is already possible to realize systems that can produce tunable delays over slightly more than one signal period. This range is sufficient for applications in analog signal processing, such as the generation of true time delays for microwave photonic applications and in particular for phased-array antennas, where the fast all-optical tunability of fibre-based delay lines is very attractive. This good match with analog signals is further supported by the intrinsic linear response of SBS amplification, as linearity is another essential requirement for analog systems $s^{57}$.

However, delay is only one aspect of slow light, and so far very few applications have taken advantage of the fact that light actually propagates slowly. Several studies have pointed out that a reduced velocity will have a significant impact on light-matter interaction. For instance, it has been predicted that the efficiency of nonlinear optical effects must scale as the slowing factor squared ${ }^{58-61}$. In practice, this means that transformations and functions based on optical nonlinearities could be realized in much shorter distances, for instance, $1 \mathrm{~cm}$ instead of $1 \mathrm{~m}$ if the light is slowed down to a tenth of its normal group velocity. However, no decisive experimental verifications have supported these predictions so far. It has also been anticipated that the sensitivity of interferometers or sensing systems can be significantly improved by reducing the speed of the light signal ${ }^{62-66}$. Amazing and unexpected applications of slow light will probably be discovered in the coming years through the use of innovative and creative solutions.

\section{doi:10.1038/nphoton.2008.94}

\section{References}

1. Krauss, T. Why do we need slow light? Nature Photon. 2, 448-450 (2008).

2. Baba, T. Slow light in photonic crystals. Nature Photon. 2, 465-473 (2008).

3. Hau, L. V., Harris, S. E., Dutton, Z. \& Behroozi, C. H. Light speed reduction to 17 metres per second in an ultracold atomic gas. Nature 397, 594-598 (1999).

4. Bigelow, M. S., Lepeshkin, N. N. \& Boyd, R. W. Superluminal and slow light propagation in a room temperature solid. Science 301, 200-202 (2003).

5. Boyd, R. W. \& Gauthier, D. J. in Progress in Optics Vol. 43 497-530 (Elsevier, Holland, 2002).

6. Xia, F., Sekaric, L. \& Vlasov, Y. Ultracompact optical buffers on a silicon chip. Nature Photon. 1, 65-71 (2007)

7. Boyd, R. W., Gauthier, D. J., Gaeta, A. L. \& Willner, A. E. Maximum time delay achievable on propagation through a slow-light medium. Phys. Rev. A 71, 059903 (2005). 
8. Khurgin, J. B. Performance limits of delay lines based on optical amplifiers. Opt. Lett. 31, 948-950 (2006).

9. Okawachi, Y. et al. Tunable all-optical delays via Brillouin slow light in an optical fiber. Phys. Rev. Lett. 94, 153902 (2005).

10. Harris, S. E. Electromagnetically induced transparency. Phys. Today 50, 36-42 (1997).

11. Boyd, R. W. Nonlinear Optics (Academic, New York, 2003).

12. Nikles, M., Thévenaz, L. \& Robert, P. A. Brillouin gain spectrum characterization in single-mode optical fibers. J. Lightwave Technol. 15, 1842-1851 (1997).

13. Agrawal, G. P. Nonlinear Fiber Optics (Academic, San Diego, 2006).

14. Mok, J. T. \& Eggleton, B. J. Expect more delays. Nature 433, 811-812 (2005).

15. Song, K. Y., González Herráez, M. \& Thévenaz, L. Observation of pulse delaying and advancement in optical fibers using stimulated Brillouin scattering. Opt. Express 13, 82-88 (2005).

16. Song, K. Y., González-Herráez, M. \& Thévenaz, L. Long optically controlled delays in optical fibers. Opt. Lett. 30, 1782-1784 (2005).

17. Zhu, Z. et al. Numerical study of all-optical slow-light delays via stimulated Brillouin scattering in an optical fiber. J. Opt. Soc. Am. B 22, 2378-2384 (2005).

18. González-Herráez, M., Song, K. Y. \& Thévenaz, L. Optically controlled slow and fast light in optical fibers using stimulated Brillouin scattering. Appl. Phys. Lett. 87, 081113 (2005).

19. Brillouin, L. Über die Fortpflanzung des Lichtes in dispergierenden Medien. Ann. Phys. 44, 203-240 (1914).

20. Brillouin, L. Wave Propagation and Group Velocity (Academic, New York, 1960).

21. Stenner, M. D., Gauthier, D. J. \& Neifeld, M. A. The speed of information in a 'fast-light' optical medium. Nature 425, 695-698 (2003).

22. Jauregui Misas, C., Petropoulos, P. \& Richardson, D. J. Slowing of pulses to $\mathrm{c} / 10$ with subwatt power levels and low latency using Brillouin amplification in a bismuth-oxide optical fiber. J. Lightwave Technol. 25, 216-221 (2007).

23. Florea, C. et al. Stimulated Brillouin scattering in single-mode $\mathrm{As}_{2} \mathrm{~S}_{3}$ and $\mathrm{As}_{2} \mathrm{Se}_{3}$ chalcogenide fibers. Opt. Express 14, 12063-12070 (2006).

24. Song, K. Y. et al. Highly efficient Brillouin slow and fast light using $\mathrm{As}_{2} \mathrm{Se}_{3}$ chalcogenide fiber. Opt. Express 14, 5860-5865 (2006).

25. Sharping, J. E., Okawachi, Y. \& Gaeta, A. L. Wide bandwidth slow light using a Raman fiber amplifier. Opt. Express 13, 6092-6098 (2005).

26. Dahan, D. \& Eisenstein, G. Tunable all optical delay via slow and fast light propagation in a Raman assisted fiber optical parametric amplifier: A route to all optical buffering. Opt. Express 13, 6234-6249 (2005)

27. Shumakher, E. et al. Large tunable delay with low distortion of $10 \mathrm{Gbit} / \mathrm{s}$ data in a slow light system based on narrow band fiber parametric amplification. Opt. Express 14, 8540-8545 (2006).

28. Schweinsberg, A. et al. Observation of superluminal and slow light propagation in erbium-doped optical fiber. Europhys. Lett. 73, 218-224 (2006).

29. Gehring, G. M. et al. Observation of backward pulse propagation through a medium with a negative group velocity. Science 312, 895-897 (2006).

30. Mok, J. T., de Sterke, C. M. \& Eggleton, B. J. Delay-tunable gap-soliton-based slow-light system. Opt. Express 14, 11987-11996 (2006).

31. Song, K. Y., González Herráez, M. \& Thévenaz, L. Gain-assisted pulse advancement using single and double Brillouin gain peaks in optical fibers. Opt. Express 13, 9758-9765 (2005).

32. Stenner, M. D. et al. Distortion management in slow-light pulse delay. Opt. Express 13, 9995-10002 (2005)

33. Zhu, Z. M. \& Gauthier, D. J. Nearly transparent SBS slow light in an optical fiber. Opt. Express 14, 7238-7245 (2006).

34. Minardo, A., Bernini, R. \& Zeni, L. Low distortion Brillouin slow light in optical fibers using AM modulation. Opt. Express 14, 5866-5876 (2006).

35. Shi, Z. et al. Design of a tunable time-delay element using multiple gain lines for increased fractional delay with high data fidelity. Opt. Lett. 32, 1986-1988 (2007).

36. González Herráez, M., Song, K. Y. \& Thévenaz, L. Arbitrary-bandwidth Brillouin slow light in optical fibers. Opt. Express 14, 1395-1400 (2006).

37. Zhu, Z. M. et al. Broadband SBS slow light in an optical fiber. J. Lightwave Technol. 25, 201-206 (2007)

38. Zadok, A., Eyal, A. \& Tur, M. Extended delay of broadband signals in stimulated Brillouin scattering slow light using synthesized pump chirp. Opt. Express 14, 8498-8505 (2006)

39. Pant, R., Stenner, M. D., Neifeld, M. A. \& Gauthier, D. J. Optimal pump profile designs for broadband SBS slow-light systems. Opt. Express 16, 2764-2777 (2008).
40. Lu, Z., Dong, Y. \& Li, Q. Slow light in multi-line Brillouin gain spectrum. Opt. Express 15, 1871-1877 (2007)

41. Yi, L. et al. Improved slow-light performance of $10 \mathrm{~Gb} / \mathrm{s} \mathrm{NRZ,} \mathrm{PSBT} \mathrm{and} \mathrm{DPSK} \mathrm{signals} \mathrm{in}$ fiberbroadband SBS. Opt. Express 15, 16972-16979 (2007).

42. Jáuregui, C., Petropoulos, P. \& Richardson, D. J. in Optical Fiber Communication Conference, San Diego, California, OSA Technical Digest (CD) OMH3 (Optical Society of America, 2008).

43. Song, K. Y. \& Hotate, K. $25 \mathrm{GHz}$ bandwidth Brillouin slow light in optical fibers. Opt. Lett. 32, 217-219 (2007)

44. Chin, S., González Herráez, M. \& Thévenaz, L. Zero-gain slow \& fast light propagation in an optical fiber. Opt. Express 14, 10684-10692 (2006)

45. Schneider, T., Henker, R., Lauterbach, K. U. \& Junker, M. Comparison of delay enhancement mechanisms for SBS-based slow light systems. Opt. Express 15, 9606-9613 (2007).

46. Chin, S. H., González Herráez, M. \& Thévenaz, L. Self-induced fast light propagation in an optical fiber based on Brillouin scattering. Opt. Express (in the press).

47. Tucker, R. S., Ku, P. C. \& Chang-Hasnain, C. J. Slow-light optical buffers: Capabilities and fundamental limitations. J. Lightwave Technol. 23, 4046-4066 (2005).

48. Khurgin, J. B. Power dissipation in slow light devices: A comparative analysis. Opt. Lett. 32, 163-165 (2007)

49. Khurgin, J. B. in Optical Amplifiers and Their Applications/Coherent Optical Technologies and Applications, Anaheim, California, OSA Technical Digest (CD) CThD3 (Optical Society of America, 2006)

50. Khurgin, J. B. Optical buffers based on slow light in electromagnetically induced transparent media and coupled resonator structures: Comparative analysis. J. Opt. Soc. Am. B 22, 1062-1074 (2005).

51. Boyd, R. W., Gauthier, D. J. \& Gaeta, A. L. Applications of slow light in telecommunications. Opt. Photon. News 17, 18-23 (2006).

52. Sharping, J. et al. All-optical, wavelength and bandwidth preserving, pulse delay based on parametric wavelength conversion and dispersion. Opt. Express 13, 7872-7877 (2005)

53. Okawachi, Y., Sharping, J. E., Xu, C. \& Gaeta, A. L. Large tunable optical delays via self-phase modulation and dispersion. Opt. Express 14, 12022-12027 (2006).

54. Okawachi, Y., Salem, R. \& Gaeta, A. L. Continuous tunable delays at $10-\mathrm{Gb} / \mathrm{s}$ data rates using self-phase modulation and dispersion. J. Lightwave Technol. 25, 3710-3715 (2007).

55. Zhu, Z. M., Gauthier, D. J. \& Boyd, R. W. Stored light in an optical fiber via stimulated Brillouin scattering. Science 318, 1748-1750 (2007)

56. Zhang, B. et al. Continuously-tunable, bit-rate variable OTDM using broadband SBS slow-light delay line. Opt. Express 15, 8317-8322 (2007).

57. Capmany, J. \& Novak, D. Microwave photonics combines two worlds. Nature Photon. 1, 319-330 (2007)

58. Soljačić, M. et al. Photonic-crystal slow-light enhancement of nonlinear phase sensitivity J. Opt. Soc. Am. B 19, 2052-2059 (2002).

59. Soljačić, M. \& Joannopoulos, J. D. Enhancement of nonlinear effects using photonic crystals. Nature Mater. 3, 211-219 (2004)

60. Matsko, A. B., Rostovtsev, Y. V., Cummins, H. Z. \& Scully, M. O. Using slow light to enhance acousto-optical effects: Application to squeezed light. Phys. Rev. Lett. 84, 5752-5755 (2000).

61. Kash, M. M. et al. Ultraslow group velocity and enhanced nonlinear optical effects in a coherently driven hot atomic gas. Phys. Rev. Lett. 82, 5229-5232 (1999).

62. Shi, Z. et al. Slow-light Fourier transform interferometer. Phys. Rev. Lett. 99, 240801 (2007).

63. Shi, Z. M., Boyd, R. W., Gauthier, D. J. \& Dudley, C. C. Enhancing the spectral sensitivity of interferometers using slow-light media. Opt. Lett. 32, 915-917 (2007).

64. Terrel, M. A., Digonnet, M. J. F. \& Fan, S. H. Performance limitation of a coupled resonant optical waveguide gyroscope. J. Lightwave Technol. (in the press).

65. Digonnet, M. J. F. New technologies in fiber sensors. in CLEO/Europe and IQEC 2007 Conference Digest $\mathrm{CH}_{2}{ }_{1}$ (Optical Society of America, 2007).

66. González-Herráez, M., Esteban, O., Naranjo, F. B. \& Thévenaz, L. in Third European Workshop on Optical Fibre Sensors, Napoli, Italy, SPIE Proc. 6619, 661937-4 (SPIE, 2007).

67. Thévenaz, L., Song, K.-Y., Chin, S.-H. \& Gonzalez-Herraez, M. in IEEE Int. Symposium Workshop on Intelligent Signal Processing, Madrid, Spain (2007)

\section{Acknowledgements}

The author gratefully acknowledges the essential contributions of M. González-Herráez, K.Y. Song and

S.H. Chin through fruitful discussions and by experimental realizations, and the support of the Swiss

National Science Foundation through project 200021-109773. 